\title{
ACCURATE CALCULATION OF FUNCTIONS USED IN A MODEL OF THE NEMATIC BEHAVIOR OF SELF-ASSEMBLING SYSTEMS
}

\author{
ALAN E. BERGER
}

\begin{abstract}
An algorithm used to evaluate double sums arising in a model describing the nematic phase behavior of surfactant solutions is demonstrated to yield approximations accurate to within a tenth of a percent. When direct summation would converge slowly, an asymptotic result is employed based on a double application of the Euler-Maclaurin sum formula.
\end{abstract}

\section{INTRODUCTION}

A model formulated by Herzfeld [3] provides a description of the liquid crystalline phase behavior of various protein and surfactant solutions. This model uses a lattice description of excluded volume effects and a phenomenological description of the reversible assembly of amphiphilic molecules into rod-like and plate-like aggregates of arbitrary size, which spontaneously align at sufficiently high concentrations. The predicted state of the system is the one which minimizes the free energy functional derived from the model. Locating this minimum when plate-like aggregates are present requires evaluation of the following four functions for $0<P, \mathscr{P}, Q<1(P, \mathscr{P}$ and $Q$ may be very close to 1 ):

$$
\begin{aligned}
G(P, \mathscr{P}, Q) & =\sum_{m=0}^{\infty} \sum_{n=0}^{\infty} P^{m} \mathscr{P}^{n} Q^{m n}, \\
G_{1}(P, \mathscr{P}, Q) & =\sum_{m} \sum_{n} m P^{m} \mathscr{P}^{n} Q^{m n}, \\
G_{2}(P, \mathscr{P}, Q) & =\sum_{m} \sum_{n} n P^{m} \mathscr{P}^{n} Q^{m n}=G_{1}(\mathscr{P}, P, Q), \\
G_{3}(P, \mathscr{P}, Q) & =\sum_{m} \sum_{n} m n P^{m} \mathscr{P}^{n} Q^{m n},
\end{aligned}
$$

cf. $[3,5,6]$. Here and below, summations whose limits are unspecified are understood to run from 0 to $\infty$. An algorithm developed by Berger and Herzfeld

Received November 4, 1988.

1980 Mathematics Subject Classification (1985 Revision). Primary 65B10, 65D15; Secondary 65B15, 82A25, 82A57.

Key words and phrases. Asymptotics, double sums, Euler-Maclaurin formula, exponential integral function, nematic, phase behavior, surfactant, self-assembling.

Research supported by the Naval Surface Warfare Center Independent Research Fund. 
for evaluating the functions in (1.1) is given in [6] and has been used to study the phase behavior of surfactant solutions $[5,6]$.

In this paper we demonstrate that the overall algorithm, assuming exact arithmetic, gives values for $G, G_{1}, G_{2}$ and $G_{3}$ with relative error (error/exact value) bounded by $1 / 1000$. While the analysis below assumes infinite precision arithmetic, the algorithm itself incorporates asymptotic expansions for certain expressions involving the exponential integral function $\operatorname{Ei}(z)$ when cancellation of terms might otherwise cause serious loss of accuracy. We next display Table 1 to indicate the sensitivity of the values of the $G$ functions to small changes in $P, \mathscr{P}$ and $Q$.

TABLE 1. Some values of the $G$ functions.

\begin{tabular}{|lllllll|}
\hline$P$ & $\mathscr{P}$ & $Q$ & $G$ & $G_{1}$ & $G_{2}$ & $G_{3}$ \\
\hline .9999 & .9999 & .999999999 & $9.16 \mathrm{E} 7$ & $8.44 \mathrm{E} 11$ & $8.44 \mathrm{E} 11$ & $7.20 \mathrm{E} 15$ \\
.9999 & .9999 & 1 & $10.0 \mathrm{E} 7$ & $10.0 \mathrm{E} 11$ & $10.0 \mathrm{E} 11$ & $10.0 \mathrm{E} 15$ \\
.9999 & .999999 & .999999999 & $2.02 \mathrm{E} 9$ & $7.98 \mathrm{E} 12$ & $7.99 \mathrm{E} 14$ & $1.22 \mathrm{E} 18$ \\
.9999 & .999999 & 1 & $10.0 \mathrm{E} 9$ & $100 . \mathrm{E} 12$ & $100 . \mathrm{E} 14$ & $100 . \mathrm{E} 18$ \\
\hline
\end{tabular}

In $\S 2$ we give a complete description of the algorithm, in preparation for the proof of accuracy which then follows.

\section{Algorithm for eVAluating (1.1)}

Let $0<P, \mathscr{P}, Q<1$. If either $P$ or $\mathscr{P}$ is less than .64 , or $Q$ is smaller than .95 , a direct partial summation is used to determine the functions in (1.1). Otherwise, a double application of the Euler-Maclaurin summation formula is employed. For direct partial summation, it is useful to rearrange the sums in (1.1) into the "herringbone pattern" $\sum_{l} T_{l}$, where $T_{l}$ designates the sum over the pairs $(m, n)$ in the half-line at and to the right of $(l, l)$ and in the half-line above $(l, l)$; e.g., for a given nonnegative integer $L$,

$$
G(P, \mathscr{P}, Q)=\sum_{l=0}^{L} H(P, \mathscr{P}, Q, l)+E(P, \mathscr{P}, Q, L),
$$

where

$$
\begin{aligned}
H(P, \mathscr{P}, Q, l) & =\sum_{m=l}^{\infty} P^{m} \mathscr{P}^{l} Q^{m l}+\sum_{n=l}^{\infty} P^{l} \mathscr{P}^{n} Q^{l n}-P^{l} \mathscr{P}^{l} Q^{l^{2}} \\
& =P^{l} \mathscr{P}^{l} Q^{l^{2}}\left[\left(1-P Q^{l}\right)^{-1}+\left(1-\mathscr{P} Q^{l}\right)^{-1}-1\right], \\
E(P, \mathscr{P}, Q, L) & =\sum_{m=L+1}^{\infty} \sum_{n=L+1}^{\infty} P^{m} \mathscr{P}^{n} Q^{m n} .
\end{aligned}
$$

Similar expressions are valid for $G_{i}, H_{i}$, and $E_{i}, i=1,2,3$. Recall that by differentiating $\sum_{i} z^{i}=(1-z)^{-1}$ and then multiplying by $z$, one has $\sum_{i} i z^{i}=z(1-z)^{-2}$ (further iterations of this procedure are used below), and so the $H_{i}$ can be easily evaluated in closed form. 
The approximate value for $G$ is the first term on the right side of (2.1a). We next indicate how to choose $L$ so that $E$ will be as small as required. Define

$$
K=L+1, \quad \rho=\rho(L)=P^{K} \mathscr{P}^{K} Q^{K^{2}}
$$

and observe that

$$
\begin{aligned}
E(P, \mathscr{P}, Q, L) & =\rho \sum_{m=K}^{\infty} \sum_{n=K}^{\infty}\left(P Q^{K}\right)^{m-K}\left(\mathscr{P} Q^{K}\right)^{n-K} Q^{(m-K)(n-K)} \\
& =\rho \sum_{i} \sum_{j}\left(P Q^{K}\right)^{i}\left(\mathscr{P} Q^{K}\right)^{j} Q^{i j}=\rho G\left(P Q^{K}, \mathscr{P} Q^{K}, Q\right) .
\end{aligned}
$$

Since $G$ is an increasing function of $P, \mathscr{P}$ and $Q, G\left(P Q^{K}, \mathscr{P} Q^{K}, Q\right)<$ $G(P, \mathscr{P}, Q)$, and so the relative error $E(P, \mathscr{P}, Q, L) / G(P, \mathscr{P}, Q)$ is bounded by $\rho$. For $P<.64$ or $\mathscr{P}<.64$ or $Q<.95$, this may be made quite small (e.g., smaller than $1 / 1000)$ without having to take $L$ very large. Note that $\rho(l)$ occurs in $H(P, \mathscr{P}, Q, l+1)$, so there is essentially no additional cost in computing the current value of $\rho$ as $l$ is successively increased until $\rho$ satisfies a given stopping criterion.

In similar fashion one has

$$
\begin{aligned}
G_{i}(P, \mathscr{P}, Q)= & \sum_{l=0}^{L} H_{i}(P, \mathscr{P}, Q, l)+K \rho G\left(P Q^{K}, \mathscr{P} Q^{K}, Q\right) \\
& +\rho G_{i}\left(P Q^{K}, \mathscr{P} Q^{K}, Q\right), \quad \text { for } i=1,2 .
\end{aligned}
$$

To evaluate $G_{1}(P, \mathscr{P}, Q)$, we initially calculate the value of the first term on the right side of $(2.4)$, with $L$ the first integer for which $\rho$ is less than or equal to half of whatever relative error tolerance $\tau>0$ is prescribed. The value of the second term on the right side of $(2.4)$ is then added in, with $G\left(P Q^{K}, \mathscr{P} Q^{K}, Q\right)$ calculated to within a relative error of $\tau / 2$ as described above. The same procedure is used for $G_{2}$. Finally, for $G_{3}$ one has

$$
\begin{aligned}
G_{3}(P, \mathscr{P}, Q)= & \sum_{l=0}^{L} H_{3}(P, \mathscr{P}, Q, l)+K^{2} \rho G\left(P Q^{K}, \mathscr{P} Q^{K}, Q\right) \\
& +K \rho G_{1}\left(P Q^{K}, \mathscr{P} Q^{K}, Q\right)+K \rho G_{2}\left(P Q^{K}, \mathscr{P} Q^{K}, Q\right) \\
& +\rho G_{3}\left(P Q^{K}, \mathscr{P} Q^{K}, Q\right) .
\end{aligned}
$$

To compute $G_{3}$ using (2.5), $L$ is the first integer for which $\rho \leq \tau / 2$, and then $G, G_{1}$ and $G_{2}$ at $\left(P Q^{K}, \mathscr{P} Q^{K}, Q\right)$ are obtained to within a relative error of $\tau / 2$ as described above.

As $P, \mathscr{P}$ and $Q$ all approach 1, these "herringbone" sums require an increasingly large number of terms. Therefore, when

$$
P \geq .64, \quad \mathscr{P} \geq .64 \quad \text { and } \quad Q \geq .95,
$$

we employ the Euler-Maclaurin summation formula (with 3 correction terms) as described below. The selection of the constants .64 and .95 in (2.6) (while somewhat arbitrary) was guided by the error analysis which follows. 
2.1. Use of the Euler-Maclaurin formula. When (2.6) obtains, we will make repeated use of the summation formula

$$
\sum_{k=0}^{\infty} f(k)=\int_{0}^{\infty} f(s) d s+\frac{f(0)}{2}-\frac{f^{(1)}(0)}{12}+\frac{f^{(3)}(0)}{720}+\tilde{r}
$$

where $f(s)$ is any "well-behaved" function on $[0, \infty)$ and $\tilde{r}$ denotes the remainder term, see, e.g., [4]. Analysis of the remainder terms when using (2.7) to evaluate (1.1) (confirmed by numerical experiments) indicates that in general it is essential to arrange to apply $(2.7)$ to $(1.1)$ only at points $(P, \mathscr{P}, Q)$ with

$$
\ln Q / \ln P \leq 1 / 4 \quad \text { and } \quad \ln Q / \ln \mathscr{P} \leq 1 / 4 \text {. }
$$

This is easily accomplished by always doing preliminary herringbone sums with $L=3$ ( $K \equiv L+1=4)$ and using equations (2.1), (2.3), (2.4) and (2.5) to reduce evaluation of $G, G_{1}, G_{2}$ and $G_{3}$ at $(P, \mathscr{P}, Q)$ to evaluation at $(\tilde{P}, \tilde{\mathscr{P}}, Q)=\left(P Q^{4}, \mathscr{P} Q^{4}, Q\right)$ for which $(2.8)$ is obviously valid. If either $P Q^{4}$ or $\mathscr{P} Q^{4}$ is less than .64 , herringbone sums are used to obtain the $G$ functions at $(\tilde{P}, \tilde{\mathscr{P}}, Q)$, otherwise $(2.7)$ is employed, as we now describe. For simplicity in the notation, we drop the tilde over $P$ and $\mathscr{P}$ when it should be clear from the context whether the original $(P, \mathscr{P}, Q)$ point or $(\tilde{P}, \tilde{\mathscr{P}}, Q)$ is under consideration (in particular it is to be understood that (2.7) is only applied to $(\tilde{P}, \tilde{\mathscr{P}}, Q))$.

Assume (2.6) and (2.8) are valid, and use the convention that sums and integrals whose limits are unspecified are understood to run from 0 to $\infty$. To determine $G(P, \mathscr{P}, Q)$ using the Euler-Maclaurin formula, define

$$
f(x, y)=P^{x} \mathscr{P}^{y} Q^{x y}=\exp (x \ln P+y \ln \mathscr{P}+x y \ln Q)
$$

and apply (2.7) to $f$ considered as a function of $x$, obtaining

$$
\begin{aligned}
\sum_{m} P^{m} \mathscr{P}^{y} Q^{m y}= & \int f(x, y) d x+\mathscr{P}^{y} / 2-(\ln P+y \ln Q) \mathscr{P}^{y} / 12 \\
& +(\ln P+y \ln Q)^{3} \mathscr{P}^{y} / 720+r(y) \quad \text { for } y \geq 0 .
\end{aligned}
$$

Bounds on the remainder $r$ are obtained in the next section. Now sum both sides of $(2.10)$ over $y=0,1,2, \ldots$ and get

$$
G(P, \mathscr{P}, Q)=\sum_{n} \int f(x, n) d x+A+\sum_{n} r(n),
$$

where $A$ denotes terms which are evaluated in closed form after straightforward albeit lengthy algebra. Since $f(x, y)>0$ and the relevant series and integrals are convergent, we have

$$
\sum_{n} \int f(x, n) d x=\int \sum_{n} f(x, n) d x .
$$


Now apply (2.7) to the right side of $(2.12)$ to find

$$
\begin{aligned}
\sum_{n} \int f(x, n) d x= & \iint f(x, y) d y d x \\
& +\int\left[f(x, 0) / 2-f_{y}(x, 0) / 12+f_{y y y}(x, 0) / 720\right] d x \\
& +\int s(x) d x
\end{aligned}
$$

where $s$ is the remainder term. Let

$$
I(P, \mathscr{P}, Q) \equiv \iint f(x, y) d y d x
$$

and let $B$ denote the second summand on the right side of (2.13), which is evaluated in closed form. We then have

$$
G(P, \mathscr{P}, Q)=I+A+B+\sum_{n} r(n)+\int s(x) d x .
$$

We approximate $G$ by $I+A+B$ (the calculation of $I$ is described immediately below), and in the next section we show that the remainder terms in (2.15) lead to a relative error of at most $1 / 1000$.

The same approach leads to

$$
G_{i}(P, \mathscr{P}, Q)=I_{i}+A_{i}+B_{i}+\sum_{n} r_{i}(n)+\int s_{i}(x) d x \quad \text { for } i=1,2,3
$$

with the notation corresponding to that in (2.15) ( $A_{i}$ and $B_{i}$ are evaluated in closed form and $r_{i}$ and $s_{i}$ are remainder terms) and where

$$
\begin{aligned}
I_{1}(P, \mathscr{P}, Q) & \equiv \iint x f(x, y) d x d y=P I_{P}(P, \mathscr{P}, Q), \\
I_{2}(P, \mathscr{P}, Q) & \equiv \iint y f(x, y) d x d y=\mathscr{P} I_{\mathcal{\rho}}(P, \mathscr{P}, Q), \\
I_{3}(P, \mathscr{P}, Q) & \equiv \iint x y f(x, y) d x d y=Q I_{Q}(P, \mathscr{P}, Q),
\end{aligned}
$$

with $f$ as in (2.9) and with $I_{P}$ denoting the partial derivative of $I$ with respect to $P$ etc. The last equality in each line of $(2.17)$ is justified by taking difference quotients approximating $I_{P}, I_{,}, I_{Q}$ and using the mean value theorem and then the Lebesgue dominated convergence theorem. The complete algebraic expressions for $A+B, A_{1}+B_{1}$, and $A_{3}+B_{3}$ are given in the Appendix and in the listing of the computer program SUM2D (available from the author) which implements the algorithm for evaluating $G, G_{1}, G_{2}$ and $G_{3}$. In the program, when $(2.16)$ is being applied, $G_{2}(P, \mathscr{P}, Q)=G_{1}(\mathscr{P}, P, Q)$ is actually obtained by calculating the approximate value of $G_{1}(\mathscr{P}, P, Q)$ via (2.16). 
2.2. Evaluation of $I, I_{1}, I_{2}$ and $I_{3}$. We have

$$
\begin{aligned}
I(P, \mathscr{P}, Q) & =\int_{y} \exp (y \ln \mathscr{P})\left\{\int_{x} \exp [(\ln P+y \ln Q) x] d x\right\} d y \\
& =\int-(\ln P+y \ln Q)^{-1} \exp (y \ln \mathscr{P}) d y .
\end{aligned}
$$

Now set

$$
\lambda=\ln P \ln \mathscr{P} / \ln Q
$$

and use the change of variables $s=\lambda+y \ln \mathscr{P}$ in (2.18) to find

$$
\begin{aligned}
I(P, \mathscr{P}, Q) & =\int_{-\infty}^{\lambda}\left(e^{-\lambda} / \ln Q\right) e^{s} s^{-1} d s \\
& =\left(e^{-\lambda} / \ln Q\right) \operatorname{Ei}(\lambda)=\lambda e^{-\lambda} \operatorname{Ei}(\lambda) /(\ln P \ln \mathscr{P}),
\end{aligned}
$$

where

$$
\operatorname{Ei}(z)=\int_{-\infty}^{z} t^{-1} \exp (t) d t \quad \text { for } z<0
$$

is the exponential integral function (cf. formula 8.211 of [2], and page 228 of [1] where $\mathrm{E}_{1}(w)=-\operatorname{Ei}(-w)$ for $\left.w>0\right)$. From (2.20) and (2.17) we have

$$
\begin{aligned}
& I_{1}(P, \mathscr{P}, Q)=\{-I(P, \mathscr{P}, Q) \ln \mathscr{P}+1 / \ln P\} / \ln Q, \\
& I_{2}(P, \mathscr{P}, Q)=\{-I(P, \mathscr{P}, Q) \ln P+1 / \ln \mathscr{P}\} / \ln Q, \\
& I_{3}(P, \mathscr{P}, Q)=\{I(P, \mathscr{P}, Q) \lambda-I(P, \mathscr{P}, Q)-1 / \ln Q\} / \ln Q .
\end{aligned}
$$

For $-1<\lambda<0$, the formula 5.1 .53 on page 231 of [1] is used to obtain $\operatorname{Ei}(\lambda)$ and thereafter $I, I_{1}, I_{2}$ and $I_{3}$. For $-59<\lambda \leq-1$ formula 5.1 .56 of [1] is used to evaluate $\lambda \exp (-\lambda) \operatorname{Ei}(\lambda)$ and thereby $I, I_{1}, I_{2}$ and $I_{3}(\operatorname{Ei}(\lambda)$ and $\exp (-\lambda) \operatorname{Ei}(\lambda)$ may be obtained using a special function library routine, if available, e.g., MMDEI in IMSL). In order to avoid serious loss of significant digits from cancellation of terms in (2.22) (particularly in $I_{3}$ ) as $-\lambda$ becomes large, for $\lambda \leq-59$ we use the asymptotic expansion for $\lambda \exp (-\lambda) \operatorname{Ei}(\lambda)$ coming from formula 8.215 of [2] (note there the $\exp (-x)$ factor also is to apply to $\left.R_{n}\right)$, viz.

$$
\lambda e^{-\lambda} \operatorname{Ei}(\lambda)=\sum_{k=0}^{n-1} \frac{k !}{\lambda^{k}}+E_{n}, \quad \text { where }\left|E_{n}\right| \leq n ! /|\lambda|^{n} .
$$

From $(2.23),(2.20)$ and (2.22) one can obtain the following approximations to be used for $\lambda \leq-59$ (cf. $\S 3.6)$ :

$$
\begin{aligned}
& I(P, \mathscr{P}, Q) \approx\left(1+1 / \lambda+2 / \lambda^{2}+6 / \lambda^{3}\right) /(\ln P \ln \mathscr{P}), \\
& I_{1}(P, \mathscr{P}, Q) \approx D /\left[(\ln P)^{2} \ln \mathscr{P}\right], \\
& \quad \text { where } D \equiv-1-2 / \lambda-6 / \lambda^{2}-24 / \lambda^{3}, \\
& I_{2}(P, \mathscr{P}, Q) \approx D /\left[(\ln \mathscr{P})^{2} \ln P\right], \\
& I_{3}(P, \mathscr{P}, Q) \approx\left(1+4 / \lambda+18 / \lambda^{2}+96 / \lambda^{3}\right) /(\ln P \ln \mathscr{P})^{2} .
\end{aligned}
$$


In the next section we prove

Theorem 2.1. The algorithm described in this section (with $\tau=1 / 1000$ when herringbone sums are used) gives values for $G, G_{1}, G_{2}$ and $G_{3}$ with relative error (error/exact value) no larger than $1 / 1000$.

It should be pointed out that the above result assumes there is no round-off error in the calculations. The expansions in (2.24) deal with the situation where it was seen that finite precision arithmetic threatened to introduce significant errors. Note, however, that we are not claiming to treat all the limitations of finite machine precision. In particular, there would be computational difficulties if the arithmetic $z=1-P$, or $z=1-\mathscr{P}$, or $z=1-Q$ loses "too many" significant digits. Error estimates which are sharper than those stated in Theorem 2.1 are given in Lemma 3.5 in $\S 3.3$, and in $\S 3.5$.

Values of $G, G_{1}, G_{2}$ and $G_{3}$ from SUM2D on test cases with $P \geq .64$, $\mathscr{P} \geq .64, Q \geq .95$ were consistent to within $0.1 \%$ with those obtained by "brute force" use of the herringbone sum option (with $\tau=1 / 10000$ ) in SUM2D.

\section{Proof of Theorem 2.1}

We demonstrate the accuracy claimed in Theorem 2.1 by obtaining bounds for: the remainder terms in the Euler-Maclaurin summations, the errors in the approximations used for the exponential integral function, and the errors in the asymptotic expansions used for $I, I_{1}, I_{2}$ and $I_{3}$ when $\lambda \leq-59$. We first give a bound (suitable for our specific applications) for the remainder term in the form of the Euler-Maclaurin sum formula given in (2.7).

3.1. A Bound for the remainder in (2.7). We will be using (2.7) with $f(s)$ of the form $e^{a s}$ or $s e^{a s}$ with a some negative constant. The following result, which follows directly from, e.g., pages 177-179 of [4], will serve our requirements.

Lemma 3.1. Assume $f(s)$ is in $C^{6}[0, \infty)$, the sum and integral in (2.7) are absolutely convergent, $f^{(i)}(s) \rightarrow 0$ as $s \rightarrow \infty$ for $i=1$ and 3 , and $f^{(6)}(s) \in$ $L^{1}(0, \infty)$. Then the remainder term $\tilde{r}$ in $(2.7)$ is bounded by

$$
|\tilde{r}| \leq \frac{2 B_{6}(0)}{6 !} \int_{0}^{\infty}\left|f^{(6)}(s)\right| d s
$$

where $B_{6}(x)$ is the sixth Bernoulli polynomial $\left(B_{6}(0)=1 / 42\right)$.

Proof. See problem 22 on page 179 of [4], let $m=2$, note from problems 19 and 20 that $\left|B_{6}(s)-B_{6}(0)\right| \leq 2 B_{6}(0)$, and then let $r \rightarrow \infty$.

As an immediate consequence one has

Corollary 3.2. Suppose, in addition to the hypotheses of Lemma 3.1, $f$ satisfies

$$
\int_{0}^{\infty}\left|f^{(6)}(s)\right| d s \leq C_{5}\left|f^{(5)}(0)\right| .
$$

Then

$$
|\tilde{r}| \leq 2 C_{5} \frac{B_{6}(0)}{6 !}\left|f^{(5)}(0)\right|=2 C_{5} \frac{\left|f^{(5)}(0)\right|}{30240}
$$


If $f(s)=e^{a s}$, then $f^{(6)}$ has one sign on $[0, \infty)$ and $C_{5}$ in (3.2) is 1 (in this case a sharper bound for $|\tilde{r}|$ is available, cf. [4, p. 154], but we will not require it here). We also have

Remark 3.3. If $f(s)=s e^{a s}$ with a some negative constant, then (3.2) is valid when

$$
C_{5}=\tilde{K}_{5} \equiv 1+\frac{2 e^{-6}}{5} \text {. }
$$

Proof. One may verify by induction that

$$
f^{(k)}(s)=\left(a^{k} s+k a^{k-1}\right) e^{a s} \quad \text { for } k=0,1, \ldots,
$$

and so

$$
f^{(6)}(s)=0 \quad \text { only at the point } p=-6 / a \text {. }
$$

Then

$$
\int_{0}^{\infty}\left|f^{(6)}(s)\right| d s=-\int_{0}^{p} f^{(6)}(s) d s+\int_{p}^{\infty} f^{(6)}(s) d s=f^{(5)}(0)-2 f^{(5)}(p)
$$

from which the result quickly follows.

For future use, we define

$$
K_{5}=5 \tilde{K}_{5}=5+2 e^{-6}
$$

and note that when $f(s)=s \exp (a s)$,

$$
\tilde{K}_{5}\left|f^{(5)}(0)\right|=K_{5} a^{4} .
$$

We are now in a position to estimate the remainder terms in (2.15) and (2.16).

3.2. Bounds on the Euler-Maclaurin remainders in (2.15) and (2.16). We first treat the remainder terms for $G(P, \mathscr{P}, Q)$. Recalling the notation in (2.9), (2.10), (2.13) and (2.15), and Corollary 3.2 and the sentence below it, we have

(3.9a) $|r(n)| \leq 2|\ln P+n \ln Q|^{5} \mathscr{P}^{n} / 30240 \quad$ for $n=0,1,2, \ldots$,

$$
|s(x)| \leq 2|\ln \mathscr{P}+x \ln Q|^{5} e^{x \ln P} / 30240 \quad \text { for } x \geq 0 .
$$

For convenience, we introduce the notation

$$
\delta=1 / 30240 \text {. }
$$

We next calculate a bound for $\int|s(x)| d x$, making use of the formula

$$
\int_{0}^{\infty} x^{k} e^{a x} d x=(-1)^{k+1} k ! / a^{k+1} \quad \text { for } a<0 \text { and } k=0,1,2, \ldots,
$$

see, e.g., 4.2 .55 in [1]. From $(2.6), \mathscr{P} \geq .64$, so $|\ln \mathscr{P}| \leq 1 / 2$, and setting $c=\ln Q / \ln P$, we have

$$
\begin{aligned}
\int|s(x)| d x & \leq 2 \delta \int(1 / 2+x|\ln Q|)^{5} e^{x \ln P} d x \\
& =2 \delta\left(1 / 32+5 c / 16+5 c^{2} / 2+15 c^{3}+60 c^{4}+120 c^{5}\right) /|\ln P|
\end{aligned}
$$


From $(2.8), c \leq 1 / 4$, and noting that

$$
|\ln z|=\sum_{n=1}^{\infty}(1-z)^{n} / n>1-z \quad \text { for } 0<z<1
$$

we obtain

$$
\int|s(x)| d x \leq 2 \delta(54.5 / 64) /|\ln P| \leq 2 \delta /(1-P) \leq 2 \delta G(P, \mathscr{P}, Q),
$$

with the last inequality following from $(2.1)$ with $L=0$.

We next provide a bound for $\sum_{n}|r(n)|$ by dominating this sum with an integral. For each positive integer $n,|r(n)|$ is bounded by

(3.14a) $2 \delta \int_{n-1}^{n}|\ln P+n \ln Q|^{5} \mathscr{P}^{n} d y \leq 2 \delta \int_{n-1}^{n}|\ln P+\ln Q+y \ln Q|^{5} \mathscr{P}^{y} d y$,

since the inequality is valid for the integrands when $n-1 \leq y \leq n$. Recalling (2.6) and the resulting fact that $|\ln P|+|\ln Q| \leq .44629+.05130 \leq 1 / 2,(3.14 \mathrm{a})$ implies

$$
\begin{aligned}
\sum_{n}|r(n)| & =|r(0)|+\sum_{n=1}^{\infty}|r(n)| \\
& \leq 2 \delta|\ln P|^{5}+2 \delta \int_{0}^{\infty}(1 / 2+y|\ln Q|)^{5} \mathscr{P}^{y} d y
\end{aligned}
$$

As above, the last term in (3.14b) is bounded by $2 \delta G(P, \mathscr{P}, Q)$. From (2.1), for $P$ and $\mathscr{P} \geq .64, G(P, \mathscr{P}, Q) \geq 2 / .36-1 \geq 4$. Since $|\ln P| \leq 1 / 2$, one has $|\ln P|^{5} \leq 1 / 32 \leq G(P, \mathscr{P}, Q) / 128$, and so from (3.13) and (3.14),

$$
\begin{aligned}
\sum_{n}|r(n)|+\int|s(x)| d x & \leq 4 \frac{1}{64} G(P, \mathscr{P}, Q) / 30240 \\
& \leq(.00014) G(P, \mathscr{P}, Q) .
\end{aligned}
$$

3.2.1. Bounds on $r_{1}$ and $s_{1}$. Estimates for the remainder terms in (2.16) generally follow a similar pattern. We start with $r_{1}$ and $s_{1}$. For $r_{1}$, apply (2.7) and $\S 3.1$ to $f(s)=\mathscr{P}^{n} s \exp (a s)$ with $a=\ln P+n \ln Q$, and obtain

$$
\left|r_{1}(n)\right| \leq 2 \delta K_{5}|\ln P+n \ln Q|^{4} \mathscr{P}^{n} \quad \text { for } n=0,1,2, \ldots .
$$

Analogous to (3.14),

$$
\begin{aligned}
\sum_{n}\left|r_{1}(n)\right| & \leq 2 \delta K_{5} \int|\ln P+\ln Q+y \ln Q|^{4} \mathscr{P}^{\prime \prime} d y+2 \delta K_{5}|\ln P|^{4} \\
& \leq 2 \delta K_{5} \int(1 / 2+y|\ln Q|)^{4} \mathscr{P}^{y} d y+2 \delta K_{5}|\ln P|^{4} \\
& \leq 2 \delta K_{5}(42 / 64) /|\ln \mathscr{P}|+\delta K_{5} / 8
\end{aligned}
$$


To obtain an error bound relative to $G_{1}$ we use

Lemma 3.4. One has $G_{1}(P, \mathscr{P}, Q) \geq 2 /|\ln \mathscr{P}| \geq 4$ when (2.6) and (2.8) are valid.

Proof. We have

$$
\begin{aligned}
G_{1}(P, \mathscr{P}, Q) & \geq \sum_{m=1}^{5} m P^{m} \sum_{n}\left(\mathscr{P} Q^{m}\right)^{n}=\sum_{m=1}^{5} \frac{m P^{m}}{1-\mathscr{P} Q^{m}} \\
& \geq \sum_{m=1}^{5} \frac{m(.64)^{m}}{1-\mathscr{P} Q^{m}} .
\end{aligned}
$$

Now using (2.8),

$$
1-\mathscr{P} Q^{m}<\left|\ln \left(\mathscr{P} Q^{m}\right)\right|=|\ln \mathscr{P}|+m|\ln Q| \leq|\ln \mathscr{P}|(1+m / 4),
$$

and so

$$
\left(1-\mathscr{P} Q^{m}\right)^{-1}>|\ln \mathscr{P}|^{-1} 4 /(4+m) .
$$

Substituting this in (3.18) and performing the arithmetic yields the first inequality of the lemma, and recalling that $|\ln \mathscr{P}| \leq 1 / 2$ gives the second. that

Equation (3.17) together with Lemma 3.4 and the fact that $K_{5}<5.005$ shows

$$
\begin{aligned}
\sum_{n}\left|r_{1}(n)\right| & \leq\left(\delta K_{5}(21 / 32)+\delta K_{5} / 32\right) G_{1}(P, \mathscr{P}, Q) \\
& \leq(.00012) G_{1}(P, \mathscr{P}, Q) .
\end{aligned}
$$

Turning our attention to $s_{1}(x)$,

$$
\left|s_{1}(x)\right| \leq 2 \delta|\ln \mathscr{P}+x \ln Q|^{5} x P^{x} \quad \text { for } x \geq 0,
$$

and so

$$
\int\left|s_{1}(x)\right| d x \leq 2 \delta|\ln P|^{-2}(222 / 64) .
$$

Taking the terms in (1.1b) with $n=0,1,2$ and 3, and using (3.19) gives

$$
G_{1}(P, \mathscr{P}, Q) \geq|\ln P|^{-2}(.64+.249+.105+.046) \geq|\ln P|^{-2} .
$$

Then (3.22) and (3.23) imply

$$
\int\left|s_{1}(x)\right| d x \leq G_{1}(P, \mathscr{P}, Q) \delta(111 / 16)<(.00023) G_{1}(P, \mathscr{P}, Q) .
$$

Since $G_{2}(P, \mathscr{P}, Q)$ is obtained by computing $G_{1}(\mathscr{P}, P, Q)$, the relative error bounds for the approximation of $G_{1}(P, \mathscr{P}, Q)$ apply as well to $G_{2}(P, \mathscr{P}, Q)$.

3.2.2. Bounds on $r_{3}$ and $s_{3}$. The same approach as above leads to

$$
\begin{aligned}
\sum_{n}\left|r_{3}(n)\right| & \leq 2 \delta K_{5} \int(y+1)(1 / 2+y|\ln Q|)^{4} \mathscr{P}^{y} d y \\
& \leq 2 \delta K_{5}\left(\frac{42}{64}|\ln \mathscr{P}|^{-1}+2 \frac{3}{32}(\ln \mathscr{P})^{-2}\right)
\end{aligned}
$$




$$
\int\left|s_{3}(x)\right| d x \leq 2 \delta K_{5} \int x(1 / 2+x|\ln Q|)^{4} P^{x} d x \leq 2 \delta K_{5}\left(2 \frac{3}{32}(\ln P)^{-2}\right) .
$$

We now show how the above estimates lead to bounds on the relative error in the values for the $G$ functions.

3.3. Consequences of the bounds on the Euler-Maclaurin remainder terms. Recall the discussion between (2.8) and (2.9). The estimates we have obtained so far enable us to demonstrate

Lemma 3.5. Assume both $(P, \mathscr{P}, Q)$ and $(\tilde{P}, \tilde{P}, Q)$ satisfy (2.6), in which case the Euler-Maclaurin formula is used to obtain the $G$ functions at $(\tilde{P}, \tilde{P}, Q)$. Assume there is no error in the evaluation of the terms $I, I_{1}, I_{2}$ and $I_{3}$ (i.e., all the error comes from the Euler-Maclaurin remainder terms). Then the relative error in the calculated value of $G(P, \mathscr{P}, Q)$ is at most .00014 , the relative error in the calculated values for $G_{1}(P, \mathscr{P}, Q)$ and $G_{2}(P, \mathscr{P}, Q)$ is no larger than .00035 , and .0005002 bounds the relative error in the computed value of $G_{3}(P, \mathscr{P}, Q)$.

Proof. Note the basic facts that: if the relative error in an approximation $v$ to some value $V$ is $\tau$, and $c$ is a constant, then the relative error in $c v$ approximating $c V$ is $\tau$; if the relative errors in $v_{1}, \ldots, v_{n}$ approximating the positive quantities $V_{1}, \ldots, V_{n}$ are all bounded by $\tau$, then the relative error in $v_{1}+\cdots+v_{n}$ approximating $V_{1}+\cdots+V_{n}$ is likewise bounded by $\tau$; and if $V$ is the sum of positive quantities $V_{1}$ and $V_{2}$, approximated by values $v_{1}$ (with relative error $\tau_{1}$ ) and $v_{2}$ (with error $e_{2}$ ), then the relative error in $v_{1}+v_{2} \approx V$ is bounded by $\tau_{1}+e_{2} / V$. The estimate for $G$ follows from (2.1a), (2.3), (2.15) and (3.15), and the estimate for $G_{1}$ is a consequence of (2.4), (2.16), (3.15), (3.20) and (3.24). The result for $G_{2}$ then also follows, noting the last sentence of $\S 3.2 .1$.

The bound for the error in $G_{3}$ is a bit more complex. From (2.5), (2.16) and the above, a bound for the relative error, $\tau_{3}$, in $G_{3}(P, \mathscr{P}, Q)$ is given by

$$
\tau_{3} \leq .00035+P^{4} \mathscr{P}^{4} Q^{16}\left(\sum_{n}\left|r_{3}(n)\right|+\int\left|s_{3}(x)\right| d x\right) / G_{3}(P, \mathscr{P}, Q) .
$$

Equations (3.25) and (3.26), and the fact that $|\ln \tilde{\mathscr{P}}| \leq 1 / 2$, give

$$
\begin{aligned}
\sum_{n}\left|r_{3}(n)\right|+\int\left|s_{3}(x)\right| d x & \leq 2 \delta K_{5}\left\{\left(\frac{42}{128}+2 \frac{3}{32}\right)(\ln \tilde{\mathscr{P}})^{-2}+2 \frac{3}{32}(\ln \tilde{P})^{-2}\right\} \\
& \leq .000802(\ln \tilde{\mathscr{P}})^{-2}+.0007(\ln \tilde{P})^{-2}
\end{aligned}
$$

Taking the terms in (1.1d) with $n=1,2,3$ and 4, and then using (3.12) and setting $\rho=P^{4} \mathscr{P}^{4} Q^{16}$ shows that

$$
G_{3}(P, \mathscr{P}, Q)>10 \rho\left(1-P Q^{4}\right)^{-2}=10 \rho(1-\tilde{P})^{-2}>10 \rho(\ln \tilde{P})^{-2} .
$$

Since $G_{3}$ is symmetric in $P$ and $\mathscr{P}$, it is also true that

$$
G_{3}(P, \mathscr{P}, Q)>10 P^{4} \mathscr{P}^{4} Q^{16}(1-\tilde{\mathscr{P}})^{-2}>10 P^{4} \mathscr{P}^{4} Q^{16}(\ln \tilde{\mathscr{P}})^{-2} .
$$


Then (3.27), (3.28) and (3.29) give

$$
\tau_{3} \leq .00035+.0000802+.00007=.0005002
$$

and we have the result.

3.4. Bounds on the errors in $I, I_{1}, I_{2}$ and $I_{3}$. We now obtain bounds on the contribution to the relative error in $G, G_{1}, G_{2}$ and $G_{3}$ from the errors in $I, I_{1}, I_{2}$ and $I_{3}$ in the three cases $-1<\lambda<0,-59<\lambda \leq-1$, and $\lambda \leq-59$, and thereby complete the proof of Theorem 2.1. Note from (2.22) that $I_{2}(P, \mathscr{P}, Q)=I_{1}(\mathscr{P}, P, Q)$, so results for $I_{2}$ follow immediately from those for $I_{1}$.

3.4.1. Bounds when $-1<\lambda<0$. From formula 5.1 .53 of [1], the error in $\operatorname{Ei}(z)$ is bounded by $2 \times 10^{-7}$. The function $\operatorname{Ei}(z)$ is decreasing on $(-\infty, 0)$, hence on $(-1,0),|\operatorname{Ei}(\lambda)| \geq|\operatorname{Ei}(-1)|>.21$ (for the latter see, e.g., [1 or 2]). Thus,

$$
\begin{aligned}
& \text { the error in } \operatorname{Ei}(\lambda)<\left(2 \times 10^{-7} / .21\right) .21<1 \times 10^{-6} \operatorname{Ei}(\lambda) \\
& \text { for }-1<\lambda<0 .
\end{aligned}
$$

Setting $R_{m n}=\{(x, y): m \leq x \leq m+1, n \leq y \leq n+1\}$, we also have

$$
\begin{aligned}
I(P, \mathscr{P}, Q) & =\sum_{m} \sum_{n} \int_{R_{m n}} f(x, y) d x d y \\
& \leq \sum_{m} \sum_{n} \int_{R_{m n}} P^{m} \mathscr{P}^{n} Q^{m n} d x d y=G(P, \mathscr{P}, Q) .
\end{aligned}
$$

Hence from (2.20),

$$
\text { the error in } I<1 \times 10^{-6} I \leq 1 \times 10^{-6} G \quad \text { for }-1<\lambda<0 \text {. }
$$

Bound for $I_{1}$. From (2.22), (3.33) and (2.20)

$$
\text { the error in } \left.I_{1} \leq \text { (the error in } I\right) \ln \mathscr{P} / \ln Q
$$

$$
<1 \times 10^{-6} I \ln \mathscr{P} / \ln Q \quad \text { for }-1<\lambda<0,
$$

$$
I_{1}=1 /(\ln P \ln Q)-I \ln \mathscr{P} / \ln Q=\left(1-\lambda e^{-\lambda} \operatorname{Ei}(\lambda)\right) /(\ln P \ln Q) .
$$

Since we wish to obtain a relative error bound for the error in $I_{1}$, we next extract a lower bound for $I_{1}$. From formula 8.212.4 of [2] with $\lambda=-x$ (which may be verified using the change of variable $w=\lambda+\ln t$ ) we have

$$
\lambda e^{--\lambda} \operatorname{Ei}(\lambda)=\int_{0}^{1} \frac{\lambda}{\lambda+\ln t} d t \quad \text { for } \lambda<0,
$$

and thus the facts that

$$
\lambda e^{-\lambda} \operatorname{Ei}(\lambda) \text { is a decreasing function of } \lambda \text { for } \lambda<0,
$$

From page 250 of [1], the value at $\lambda=-1$ of $\lambda e^{-\lambda} \operatorname{Ei}(\lambda)=-\lambda e^{-\lambda} \mathrm{E}_{1}(-\lambda)$ is less than .6 , so from $(3.34 b)$ and $(3.35 b)$

$$
I_{1} \geq .4 /(\ln P \ln Q) \quad \text { and } \quad I_{1}^{-1} \leq(\ln P \ln Q) / .4 \quad \text { for }-1<\lambda<0,
$$


and so

$$
\begin{aligned}
& \text { the error in } I_{1}<\left[\left(1 \times 10^{-6} I \ln \mathscr{P} / \ln Q\right) I_{1}^{-1}\right] I_{1} \\
& \quad \leq 2.5 \times 10^{-6} \lambda e^{-\lambda} \operatorname{Ei}(\lambda) I_{1} \leq 1.5 \times 10^{-6} I_{1} \quad \text { for }-1<\lambda<0 .
\end{aligned}
$$

Our bound for the error in $I_{1}$ will be completed using the following result.

Lemma 3.6. Assume (2.6) and (2.8) are valid. Then

$$
I_{1}(P, \mathscr{P}, Q)<3 G_{1}(P, \mathscr{P}, Q)
$$

Equation (3.37) and Lemma 3.6 give the estimate

$$
\text { the error in } I_{1}<4.5 \times 10^{-6} G_{1} \text { for }-1<\lambda<0 \text {. }
$$

The pattern of the demonstration of (3.38) will be repeated throughout this section.

Proof of Lemma 3.6. We have $I_{1} \leq \sum_{m} \sum_{n}(m+1) P^{m} \mathscr{P}^{n} Q^{m n}=G_{1}+G$. Now $G=(1-\mathscr{P})^{-1}+\sum_{m>0} \sum_{n} P^{m} \mathscr{P}^{n} Q^{m n}<(1-\mathscr{P})^{-1}+G_{1}$, and

$$
\begin{aligned}
|\ln z| & =\sum_{i=1}^{\infty}(1-z)^{i} / i<(1-z)+\sum_{i=2}^{\infty}(1-z)^{i} / 2 \\
& =(1-z)+\frac{(1-z)^{2}}{2 z} \quad \text { for } 0<z<1,
\end{aligned}
$$

and so

(3.39) $|\ln z| \leq(1-z)+(1-z)(.36 / 1.28) \leq 4(1-z) / 3 \quad$ for $.64 \leq z<1$.

Since (2.6) is being assumed, (3.39) implies that

$$
(1-\mathscr{P})^{-1} \leq 4|\ln \mathscr{P}|^{-1} / 3
$$

and this together with Lemma 3.4 and the above gives

$$
G(P, \mathscr{P}, Q)<2 G_{1}(P, \mathscr{P}, Q) \quad \text { when }(2.6) \text { and }(2.8) \text { are valid }
$$
and hence $I_{1}<3 G_{1}$.

Bound for $I_{3}$ when $-1<\lambda<0$. Estimating the relative error in $I_{3}$ follows the same pattern of obtaining an upper bound for the error in $I_{3}$ and a lower bound for the value of $I_{3}$. From (2.22) and (2.20),

$$
\text { the error in } \begin{aligned}
I_{3} & \leq 2(\text { the error in } \operatorname{Ei}(\lambda)) e^{-\lambda} /(\ln Q)^{2} \\
& \leq\left(4 \times 10^{-7} e(\ln Q)^{-2} I_{3}^{-1}\right) I_{3} .
\end{aligned}
$$

We next determine a lower bound for $I_{3}$, which then gives an upper limit on the size of $I_{3}^{-1}$. Again from (2.22) and (2.20),

$$
I_{3}(P, \mathscr{P}, Q)=k(\lambda) /(\ln Q)^{2} \quad \text { and thus } \quad I_{3}^{-1}=(\ln Q)^{2} / k(\lambda),
$$

where

$$
k(\lambda) \equiv \lambda e^{-\lambda} \operatorname{Ei}(\lambda)-e^{-\lambda} \operatorname{Ei}(\lambda)-1
$$


We will demonstrate below that

$$
k(\lambda)=\int_{0}^{\infty} \frac{t e^{-t}}{(t-\lambda)^{2}} d t
$$

which shows that $k(\lambda)$ is a positive increasing function for $-\infty<\lambda<0$. Thus, using, e.g., Table 5.6 on page 250 of [1],

$$
k(\lambda) \geq k(-1)>.1926 \text { for }-1<\lambda<0 .
$$

Equations (3.42), (3.43) and (3.44) give

$$
\text { the error in } I_{3} \leq\left(4 \times 10^{-7} e / .1926\right) I_{3} \leq 5.66 \times 10^{-6} I_{3} \text {. }
$$

We also prove below

Lemma 3.7. Assume (2.6) and (2.8) are valid. Then

$$
I_{3}(P, \mathscr{P}, Q)<12 G_{3}(P, \mathscr{P}, Q) \text {. }
$$

This together with (3.45) gives the estimate

the error in $I_{3}(P, \mathscr{P}, Q) \leq 6.8 \times 10^{-5} G_{3}(P, \mathscr{P}, Q) \quad$ for $-1<\lambda<0$.

Proof of (3.43c). From formula 8.212.3 of [2] (which may be verified by integration by parts and a simple change of variable),

$$
\lambda e^{-\lambda} \operatorname{Ei}(\lambda)-1=\int_{0}^{\infty} \frac{\lambda e^{-t}}{(t-\lambda)^{2}} d t
$$

Starting from (2.21) with $t$ replaced by $w$, and then using the change of variable $w=\lambda-t$,

$$
\operatorname{Ei}(\lambda)=\int_{-\infty}^{\lambda} \frac{e^{u}}{w} d w=\int_{0}^{\infty} \frac{e^{\lambda} e^{-t}}{\lambda-t} d t=\int_{0}^{\infty} \frac{(\lambda-t) e^{\lambda} e^{-t}}{(t-\lambda)^{2}} d t
$$

so

$$
-e^{-\lambda} \operatorname{Ei}(\lambda)=\int_{0}^{\infty} \frac{(t-\lambda) e^{-t}}{(t-\lambda)^{2}} d t
$$

and summing (3.47) and (3.49) gives the desired result, (3.43c).

Proof of Lemma 3.7. Bounding the integrand for $I_{3}$ over each $R_{m n}$ results in

$$
\begin{aligned}
I_{3}(P, \mathscr{P}, Q) \leq & G_{3}(P, \mathscr{P}, Q)+G_{2}(P, \mathscr{P}, Q) \\
& +G_{1}(P, \mathscr{P}, Q)+G(P, \mathscr{P}, Q) .
\end{aligned}
$$

Also,

$$
\begin{aligned}
G_{1}(P, \mathscr{P}, Q) & =P(1-P)^{-2}+\sum_{m} \sum_{n>0} m P^{m} \mathscr{P}^{n} Q^{m n} \\
& <P(1-P)^{-2}+G_{3}(P, \mathscr{P}, Q)
\end{aligned}
$$

and similarly

$$
G(P, \mathscr{P}, Q)<(1-P)^{-1}+(1-\mathscr{P})^{-1}+G_{3}(P, \mathscr{P}, Q)
$$


Performing the same type of computation as used to obtain (3.23),

$$
\begin{aligned}
G_{3}(P, \mathscr{P}, Q) & >\sum_{n=1}^{3} n \mathscr{P}^{n} \sum_{m} m\left(P Q^{n}\right)^{m}>(.249+.210+.138)|\ln P|^{-2} \\
& =.597|\ln P|^{-2} \quad \text { when (2.6) and (2.8) are valid. }
\end{aligned}
$$

Since $P \geq .64$, one has $|\ln P| \leq .44629$, and (3.53) and (3.39) with $z=P$ give

$$
G_{3}(P, \mathscr{P}, Q)>|\ln P|^{-1} .597 / .44629>4|\ln P|^{-1} / 3 \geq(1-P)^{-1} .
$$

Interchanging $P$ and $\mathscr{P}$ in (3.54) yields

$$
G_{3}(P, \mathscr{P}, Q)>(1-\mathscr{P})^{-1} \text {, }
$$

and so

$$
G(P, \mathscr{P}, Q)<3 G_{3}(P, \mathscr{P}, Q) .
$$

Also from (3.53) and (3.39) with $z=P$,

$$
3 G_{3}(P, \mathscr{P}, Q)>1.791|\ln P|^{-2}>16|\ln P|^{-2} / 9>P /(1-P)^{2},
$$

and therefore

$$
G_{1}(P, \mathscr{P}, Q)<4 G_{3}(P, \mathscr{P}, Q),
$$

and, interchanging $P$ and $\mathscr{P}$ in (3.58), $G_{2}<4 G_{3}$. Equations (3.50), (3.56) and (3.58) give the result.

3.4.2. Bounds when $-59<\lambda \leq-1$. The approach for obtaining these estimates follows a course similar to the above. From formula 5.1.56 of [1], the error $\zeta$ in $\lambda e^{-\lambda} \operatorname{Ei}(\lambda)$ is less than $2 \times 10^{-8}$ for $-59<\lambda \leq-1$, and from Table 5.6 of [1] and (3.35b), $\lambda e^{-\lambda} \operatorname{Ei}(\lambda) \geq .5963$ for $\lambda \leq-1$, hence

$$
\zeta<\left(2 \times 10^{-8} / .5963\right) \lambda e^{-\lambda} \operatorname{Ei}(\lambda) \leq 3.4 \times 10^{-8} \lambda e^{-\lambda} \operatorname{Ei}(\lambda) .
$$

Therefore, from (2.20) and (3.32),

$$
\text { the error in } I<3.4 \times 10^{-8} I \leq 3.4 \times 10^{-8} G \text {. }
$$

The error in $I_{1}$ is bounded by the error in $I$ times $\ln \mathscr{P} / \ln Q$, and

$$
I_{1}^{-1} \leq \ln P \ln Q /(1-\xi),
$$

where $\xi$ is the value of $\lambda e^{-\lambda} \operatorname{Ei}(\lambda)$ at $\lambda=-59$. We conservatively bound $\xi$ from above by the value of $\lambda e^{-\lambda} \operatorname{Ei}(\lambda)$ at $\lambda=1 / .015$ given in Table 5.2 of [1] (one could obtain a somewhat sharper bound, if desired, by using formula 5.1 .55 of [1] at $\lambda=-59)$, which leads to $(1-\xi) \geq .0145$, and so

$$
\text { the error in } \begin{aligned}
I_{1} & \leq\left(3.4 \times 10^{-8} I \ln \mathscr{P} / \ln Q\right)(\ln P \ln Q / .0145) I_{1} \\
& \leq 2.4 \times 10^{-6} I_{1} \leq 7.2 \times 10^{-6} G_{1},
\end{aligned}
$$

using $(3.35 \mathrm{c})$ and Lemma 3.6.

The error in $I_{3}$ is bounded by twice the error in $I$ times $\lambda / \ln Q$, while $I_{3}^{-1} \leq(\ln Q)^{2} / k(-59)$. Again using Table 5.2 of [1], .00021 is a conservative lower bound for $k(-59)$, and this together with $(3.60)$ and $(3.35 \mathrm{c})$ gives the error in $I_{3} \leq\left(6.8 \times 10^{-8} / .00021\right) I_{3} \leq 3.24 \times 10^{-4} I_{3}$. 
Lemma 3.7 is no longer adequate to obtain the desired result, but recall we are actually applying (3.62) to $(\tilde{P}, \tilde{P}, Q)$ in the context of $(2.5)$ (with $K=$ $4)$. Thus our next step is to bound the error in $\rho G_{3}(\tilde{P}, \tilde{P}, Q)$ in terms of $G_{3}(P, \mathscr{P}, Q)$ when $\lambda \leq-1$. From (2.16) applied at the point $(\tilde{P}, \tilde{P}, Q)$, and setting $\tilde{G}_{3}=G_{3}(\tilde{P}, \tilde{P}, Q)$ and $G_{3}=G_{3}(P, \mathscr{P}, Q)$, and similarly with $\tilde{I}_{3}$ and $I_{3}$,

the error in $\rho \tilde{G}_{3} / G_{3}=\rho$ (the error in $\left.\tilde{I}_{3}\right) / G_{3}$

$$
+\rho\left(\sum_{n} r_{3}(n)+\int s_{3}(x) d x\right) / G_{3} .
$$

Now from (3.28) and (3.29), the last term in (3.63) is bounded by .0001502, while (2.16) and inspection of the signs of the terms in the expression for $A_{3}+B_{3}$ in the Appendix gives

$$
\begin{aligned}
\tilde{G}_{3}= & \tilde{I}_{3}+\sum_{n} r_{3}(n)+\int s_{3}(x) d x \\
& -\frac{\tilde{\mathscr{P}}}{12(1-\tilde{\mathscr{P}})^{2}}-\frac{1}{12(\ln \tilde{P})^{2}}+\text { positive terms. }
\end{aligned}
$$

Again using (3.29), (3.64a) gives

$$
\rho \tilde{I}_{3} / G_{3} \leq \rho \tilde{G}_{3} / G_{3}+.0001502+1 / 60 .
$$

Since all the terms on the right side of (2.5) are positive, $\rho \tilde{G}_{3} / G_{3} \leq 1$ and

$$
\rho \tilde{I}_{3} / G_{3} \leq 1+.0001502+1 / 60 \leq 1.017 .
$$

Then $(3.62)$ at $(\tilde{P}, \tilde{P}, Q),(3.63)$ and $(3.65)$ yield

$$
\text { the error in } \rho \tilde{G}_{3} / G_{3} \leq .00032951+.0001502 \leq .00048 \text {. }
$$

3.4.3. Bounds when $\lambda \leq-59$. We first note that repeated integration by parts in (2.21) leads to (2.23), and to the fact that the sign of $E_{n}$ in (2.23) is the same as the sign of the first omitted term, viz., $n ! / \lambda^{n}$. Let $e_{0}$ be the value which, when added to the approximation for $I$ in the right side of (2.24), gives $I$ exactly, and similarly with $e_{1}$ for $I_{1}$, and $e_{3}$ for $I_{3}$. Then the same algebra which gives (2.24) shows that $e_{0}$ and $e_{1}$ have the same sign as, and are bounded by the magnitude of $24 \lambda^{-4} /(\ln P \ln \mathscr{P})$ and $-120 \lambda^{-4} /\left[(\ln P)^{2} \ln \mathscr{P}\right]$, respectively; and $e_{3}$ is the sum of two terms with the same signs as, and bounded by the magnitudes of $720 \lambda^{-4} /(\ln P \ln \mathscr{P})^{2}$ and $-120 \lambda^{-4} /(\ln P \ln \mathscr{P})^{2}$. The error in $I$, when $\lambda \leq-59$, is thus bounded by $2 \times 10^{-6} /(\ln P \ln \mathscr{P})$, while

$$
I \geq\left(1-1 / 59-6 / 59^{3}\right) /(\ln P \ln \mathscr{P}) \geq .983 /(\ln P \ln \mathscr{P}) .
$$

Thus,

$$
\text { the error in } I \leq 2.04 \times 10^{-6} I \leq 2.04 \times 10^{-6} G \text {. }
$$


Analogous calculations lead to

$$
\begin{aligned}
& \text { the error in } I_{1} \leq 1.04 \times 10^{-5} I_{1} \leq 3.12 \times 10^{-5} G_{1}, \\
& \text { the error in } I_{3} \leq 6.46 \times 10^{-5} I_{3} .
\end{aligned}
$$

Comparing (3.69b) with (3.62), we see that (3.66) is also valid for $\lambda \leq-59$.

3.5. Proof of Theorem 2.1. Assume both $(P, \mathscr{P}, Q)$ and $(\tilde{P}, \tilde{P}, Q)$ satisfy (2.6). The results and method of proof of Lemma 3.5, together with the bounds on the error in $I, I_{1}, I_{2}$ and $I_{3}$ in $\$ 3.4$ demonstrate that: the relative error in $G(P, \mathscr{P}, Q)$ is bounded by .00014204 , the relative error in $G_{1}(P, \mathscr{P}, Q)$ (and $G_{2}$ ) is bounded by .0003812 , and .0008612 bounds the relative error in $G_{3}(P, \mathscr{P}, Q)$. (We have retained extra digits in various constants to make it easier to follow the calculations of the bounds.)

3.6. Closing remarks. The value $\lambda_{a}=-59$, beyond which $(2.24)$ is used, was determined as that $\lambda$ at which rough estimates for the errors in $I_{3}$ using (2.22) and using (2.24) were equal; the estimates being, respectively,

(the error in $I) \lambda / \ln Q \approx\left(2 \times 10^{-8} /(\ln P \ln \mathscr{P})\right) \lambda / \ln Q=2 \times 10^{-8}(\ln Q)^{-2}$

and

$$
840 \lambda^{-4}(\ln P \ln \mathscr{P})^{-2}=840 \lambda^{-6}(\ln Q)^{-2} \text {. }
$$

The resulting $\lambda_{a}$ is $-58.958 \approx-59$. If the accuracy of the evaluation of $\lambda e^{-\lambda} \operatorname{Ei}(\lambda)$ is increased, a corresponding value of $\lambda_{a}$ should be used, and corresponding error estimates can be readily obtained with the methods developed in $\S 3$.

APPendix. Closed form eXPREsSions for the QuANTITIES $A+B$,

$$
A_{1}+B_{1} \text { AND } A_{3}+B_{3} \text { IN EQUATIONS }(2.15),(2.16) \text {. }
$$

$$
\begin{aligned}
A+B= & \frac{1}{2(1-\mathscr{P})}-\frac{\ln P}{12(1-\mathscr{P})}-\frac{\mathscr{P} \ln Q}{12(1-\mathscr{P})^{2}}+\frac{(\ln P)^{3}}{720(1-\mathscr{P})} \\
& +\frac{\mathscr{P}(\ln P)^{2} \ln Q}{240(1-\mathscr{P})^{2}}+\frac{\mathscr{P} \ln P(\ln Q)^{2}}{240(1-\mathscr{P})^{2}}+\frac{\mathscr{P}^{2} \ln P(\ln Q)^{2}}{120(1-\mathscr{P})^{3}} \\
& +\frac{(\ln Q)^{3}}{720}\left[\frac{\mathscr{P}}{(1-\mathscr{P})^{2}}+\frac{6 \mathscr{P}^{2}}{(1-\mathscr{P})^{3}}+\frac{6 \mathscr{P}^{3}}{(1-\mathscr{P})^{4}}\right] \\
& -\frac{1}{2 \ln P}+\frac{\ln \mathscr{P}}{12 \ln P}-\frac{\ln Q}{12(\ln P)^{2}}-\frac{(\ln \mathscr{P})^{3}}{720 \ln P} \\
& +\frac{(\ln \mathscr{P})^{2} \ln Q}{240(\ln P)^{2}}-\frac{\ln \mathscr{P}(\ln Q)^{2}}{120(\ln P)^{3}}+\frac{(\ln Q)^{3}}{120(\ln P)^{4}}
\end{aligned}
$$




$$
\begin{aligned}
A_{1}+B_{1}=- & \frac{1}{12(1-\mathscr{P})}+\frac{(\ln P)^{2}}{240(1-\mathscr{P})}+\frac{\mathscr{P} \ln P \ln Q}{120(1-\mathscr{P})^{2}}+\frac{\mathscr{P}(\ln Q)^{2}}{240(1-\mathscr{P})^{2}} \\
& +\frac{\mathscr{P}^{2}(\ln Q)^{2}}{120(1-\mathscr{P})^{3}}+\frac{1}{2(\ln P)^{2}}-\frac{\ln \mathscr{P}}{12(\ln P)^{2}}+\frac{\ln Q}{6(\ln P)^{3}} \\
& +\frac{(\ln \mathscr{P})^{3}}{720(\ln P)^{2}}-\frac{\ln Q(\ln \mathscr{P})^{2}}{120(\ln P)^{3}}+\frac{\ln \mathscr{P}(\ln Q)^{2}}{40(\ln P)^{4}}-\frac{(\ln Q)^{3}}{30(\ln P)^{5}} \\
A_{3}+B_{3}=- & \frac{\mathscr{P}}{12(1-\mathscr{P})^{2}}+\frac{\mathscr{P}(\ln P)^{2}}{240(1-\mathscr{P})^{2}}+\frac{\mathscr{P} \ln P \ln \mathscr{P}}{120(1-\mathscr{P})^{2}}+\frac{\mathscr{P}^{2} \ln P \ln Q}{60(1-\mathscr{P})^{3}} \\
& +\frac{\mathscr{P}(\ln Q)^{2}}{240(1-\mathscr{P})^{2}}+\frac{\mathscr{P}^{2}(\ln Q)^{2}}{40(1-\mathscr{P})^{3}}+\frac{\mathscr{P}^{3}(\ln Q)^{2}}{40(1-\mathscr{P})^{4}}-\frac{1}{12(\ln P)^{2}} \\
& +\frac{(\ln \mathscr{P})^{2}}{240(\ln P)^{2}}-\frac{\ln \mathscr{P} \ln Q}{60(\ln P)^{3}}+\frac{(\ln Q)^{2}}{40(\ln P)^{4}}
\end{aligned}
$$

\section{BIBLIOGRAPHY}

1. M. Abramowitz and I. A. Stegun, Handbook of mathematical functions, Dover, New York, ninth printing.

2. I. S. Gradshteyn and I. M. Ryzhik, Table of integrals, series, and products, Academic Press, New York, 1980.

3. J. Herzfeld, Liquid crystalline order in self-assembling systems: orientation dependence of the particle size distribution, J. Chem. Phys. 88 (1988), 2776-2779.

4. F. B. Hildebrand, Introduction to numerical analysis, McGraw-Hill, New York, 1956.

5. M. P. Taylor, A. E. Berger and J. Herzfeld, Theory of liquid crystalline phases in amphiphilic systems, Molecular Crystals and Liquid Crystals 157 (1988), 489-500.

6. M. P. Taylor, A. E. Berger and J. Herzfeld, Theory of amphiphilic liquid crystals: multiple phase transitions in a model micellar system, J. Chem. Phys. 91 (1989), 528-538.

Applied Mathematics Branch-Code R44, Naval Surface Warfare Center, Silver SPRING, MARYLAND 20903-5000.E-mail: aberger@nswc-wo.arpa 